\title{
Strangeness Suppression in Proton-Proton Collisions
}

\author{
Hans-Joachim Drescher ${ }^{\$}$, Jörg Aichelin ${ }^{\dagger}$, Klaus Werner $^{\dagger}$ \\ \$New York University, 4 Washington Place, New York NY 10003 \\ ${ }^{\dagger}$ SUBATECH, La Chantrerie 4, rue Alfred Kastler BP 20722 - 44307 Nantes-cedex 3
}

October 26, 2018

\begin{abstract}
We analyse strangeness production in protonproton (pp) collisions at SPS and RHIC energies, using the recently advanced NeXus approach. After having verified that the model reproduces well the existing data, we interpret the results: strangeness is suppressed in protonproton collisions at SPS energy as compared to electron-positron $\left(\mathrm{e}^{+} \mathrm{e}^{-}\right)$annihilation due to the limited masses of the strings produced in the reaction, whereas high energy pp and $\mathrm{e}^{+} \mathrm{e}^{-}$collisions agree quantitatively. Thus strangeness suppression at SPS energies is a consequence of the limited phase-space available in string fragmentation.
\end{abstract}

\section{Introduction}

Strangeness enhancement in ultra-relativistic nucleus-nucleus collisions has been proposed as a signal for the formation of a quark-gluon plasma[1]. At the SPS it has been observed for example by the WA97 [2] collaboration, and the experiments of RHIC will certainly analyse strangeness is detail. When one talks about strangeness enhancement one has first to specify the point of reference which are usually protonproton reactions. How meaningful is this point of reference? In order to answer this question, we analyse the strangeness production of protonproton collisions at different energies as compared to $\mathrm{e}^{+} \mathrm{e}^{-}$annihilation which is rather energy independent. This choice is justified by the fact that particle production seems to be universal in all kinds of elementary high energy reactions.

\section{The NEXus Model and the Role of String Fragmenta- tion}

Before we start with an analysis of the physics of multiplicities of different hadrons we explain the basic features of our approach (NEXUS) which describes simultaneously high energy electronpositron annihilation and hadron-hadron scattering. The details may be found in reference [3].

The common feature between hadron-hadron collisions and electron-positron annihilation is the creation of strings which finally produce observable hadrons. In the former case the exchange of a Pomeron leads to the formation of two strings, in the latter a string is spanned between the quark-antiquark created by the decay of a virtual photon or a Z-boson. At low energies the string just consists of two partons at the end-points, at higher energies perturbative gluons appear in initial or final state radiation which are mapped onto the string as the so-called kinks.

Once a string is created it evolves according to the Nambu-Goto Lagrangian for a classical relativistic string. In order to produce hadrons we use the area-law of Artru-Menessier. Here, the probability of the string to break is proportional to the invariant area swept over in Minkowski space. The breaking is then determined by one parameter, the break probability. If it is small, the string breaks at later times, producing less but heavier fragments and vice-verse. Flavor production is governed by two additional parameters, the probability to create a strange quarkantiquark pair (otherwise up or down pairs are created in equal fractions) and the probability to create a diquark-antidiquark pair. The former therefore governs strangeness production, the latter baryon production and the combination of both rules the creation of hyperons. The 


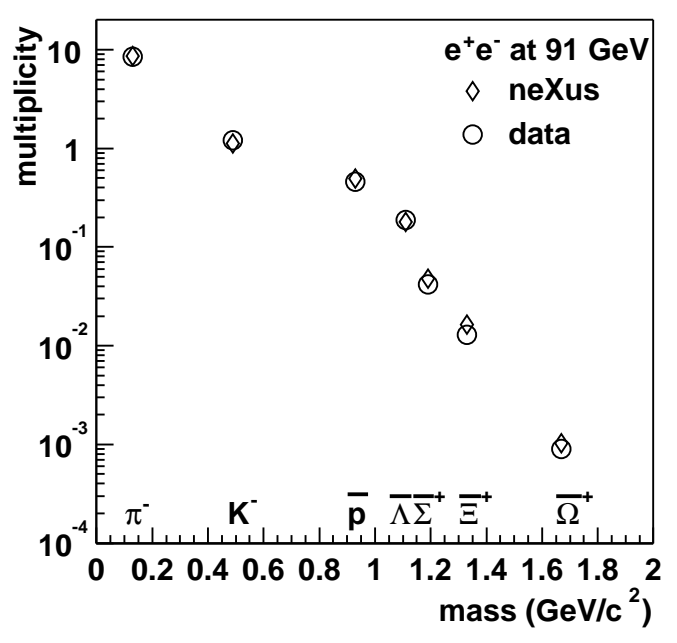

Figure 1: Results for $\mathrm{e}^{+} \mathrm{e}^{-}$annihilation at 91.2 $\mathrm{GeV}$ compared with data from the Opal collaboration [4].
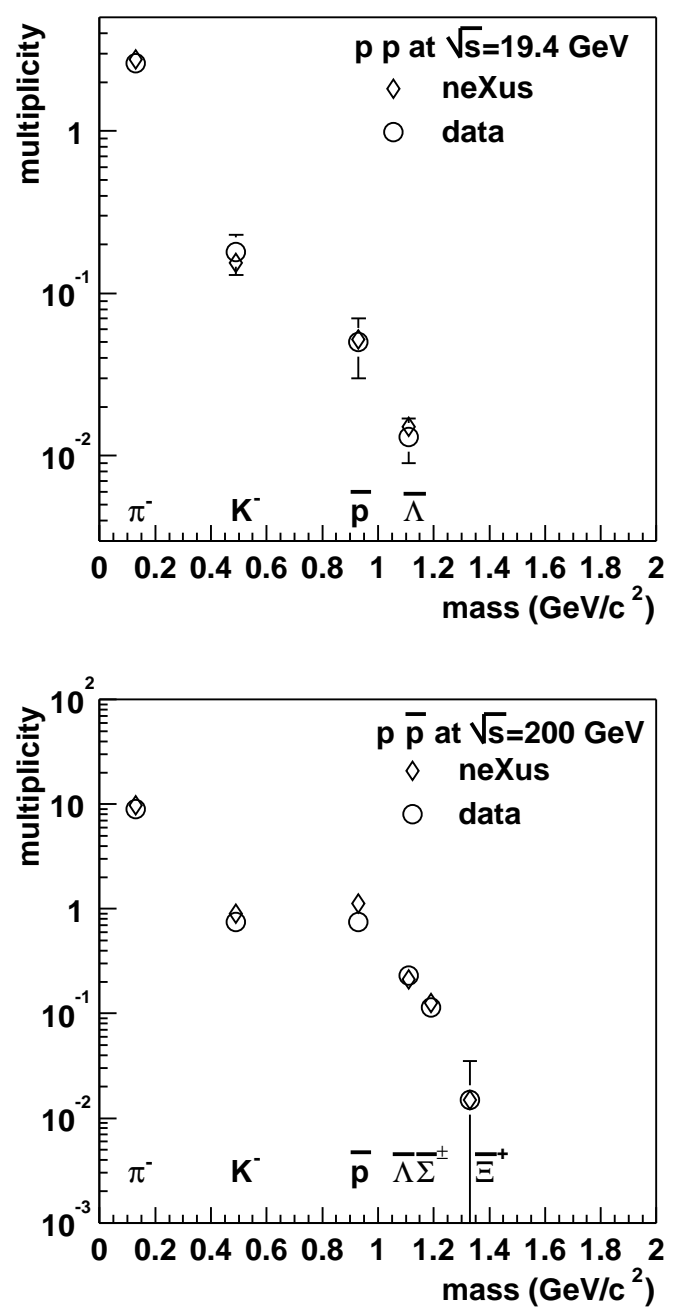

Figure 2: Comparison of the model with data for proton proton collisions at $\sqrt{s}=19.4 \mathrm{GeV}[\mathrm{f]}$ and anti-proton-proton collisions at $\sqrt{s}=200$ decay of strings can be seen as a longitudinal (one dimensional) microscopic phase space decay, therefore is is more difficult to produce heavier particles than lighter ones.

In figure 1 we show particle yields of $\mathrm{e}^{+} \mathrm{e}^{-}$annihilation from our model compared with data from the Opal collaboration 《4]. The two parameters for strangeness and diquarks have been adjusted to fit these data, and the model is capable to describe a multitude of data. One can convince oneself in reference [3] that also eventshapes and differential spectra are reproduced nicely. The same model applied to hadronhadron collisions gives the results shown in figure 2. Here we compare two energies which are close to the ones we are going to use for our analysis. Furthermore we consider only negatives or antibaryons as produced particles, results for the other particles agree in a similar way with data. We can conclude that for $e^{+} e^{-}$annihilation as well as for pp and anti-proton-proton $(\mathrm{p} \overline{\mathrm{p}})$ collisions NeXus agrees with the experimentally observed particle yields.

\section{Interpretation of Results}

We are now going to interpret the abovementioned results based on NEXUs calculations. In figure 3 we show multiplicities of particles produced in pp collisions at $17.3 \mathrm{GeV}$ (SPS) and at $200 \mathrm{GeV}$ (RHIC) as compared with $\mathrm{e}^{+} \mathrm{e}^{-}$at $91.2 \mathrm{GeV}$. To account for spin-degeneracy we divide the obtained multiplicity by the factor $2 j+1$. First of all one sees that the particle yields fall roughly exponentially with the particle mass. This is a simple phase-space effect: heavier particles are more difficult to produce. Striking is the unexpected similarity of pp at 200 $\mathrm{GeV}$ with $\mathrm{e}^{+} \mathrm{e}^{-}$at $91.2 \mathrm{GeV}$. As described above, the formation of strings is quite different in $\mathrm{pp}$ as compared to $\mathrm{e}^{+} \mathrm{e}^{-}$reactions. The spectra obtained for $17.3 \mathrm{GeV}$ is considerably steeper. We see as well very little difference between strange and non strange hadrons, all fall on a common curve.

This effect can be seen clearer in figure 4, where the multiplicities are plotted normalized to the ones of $\mathrm{e}^{+} \mathrm{e}^{-}$. The ratio for pp interactions with respect to $\mathrm{e}^{+} \mathrm{e}^{-}$at RHIC energies is close to one. Only the heaviest particle - the Omega - is slightly suppressed in pp. At SPS energies the yields for pp collisions show a completely different behavior: the ratio with respect to $\mathrm{e}^{+} \mathrm{e}^{-}$falls off strongly as a function of the mass. 


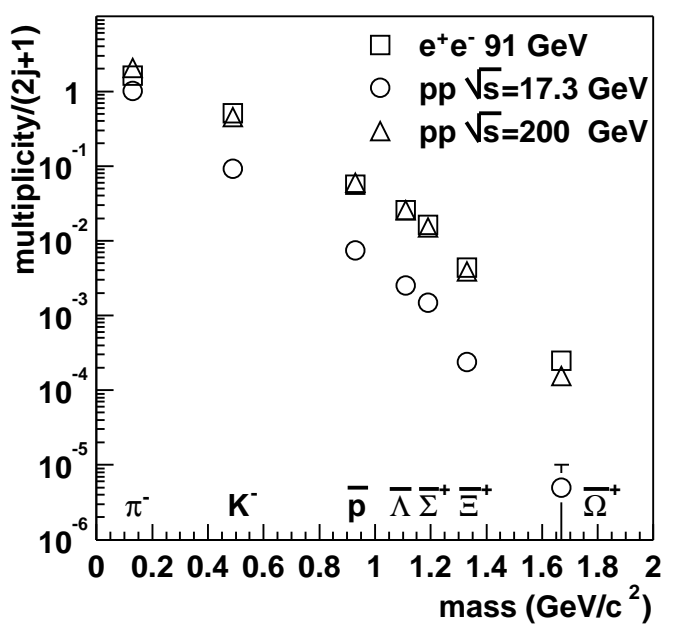

Figure 3: Particle yields of different reactions calculated with NEXUs.

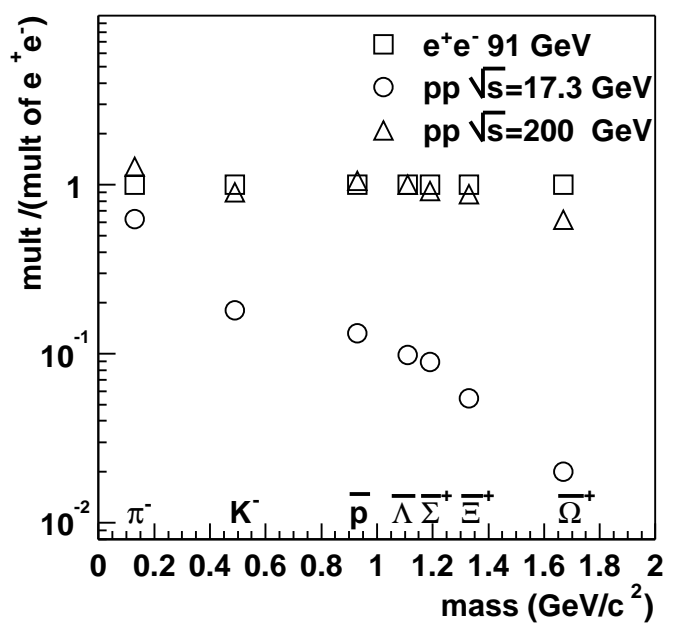

Figure 4: The multiplicities of particles normalized to $\mathrm{e}^{+} \mathrm{e}^{-}$at $91.2 \mathrm{GeV}$. Proton-proton collisions at $200 \mathrm{GeV}$ are very similar to $\mathrm{e}^{+} \mathrm{e}^{-}$, at $17.3 \mathrm{GeV}$ a suppression of heavier particles is noticeable.

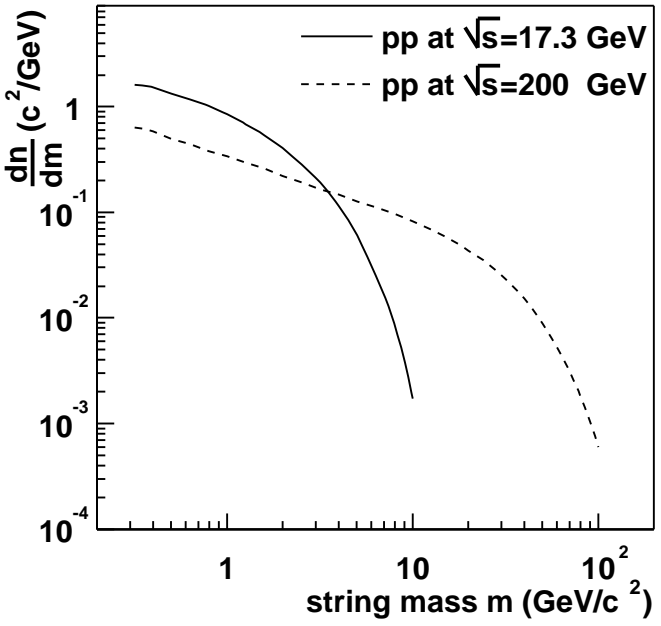

Figure 5: The distribution of string masses for two reactions pp at $17.3 \mathrm{GeV}$ and $200 \mathrm{GeV}$.

Where does this effect come from? No new physics enters between the two energies, with exception of the minijets which are more abundant at higher energies. But this influences only differential spectra like that of transverse momenta and not the relative abundance of particles.

The answer becomes quite clear when we look at the masses of the strings which finally produce the particles. Figure 5 shows the distribution $\frac{d n}{d m}$ of string masses produced at the two different energies. We leave out the case of $\mathrm{e}^{+} \mathrm{e}^{-}$ since here we have in most cases one string of mass $91.2 \mathrm{GeV}$. Only if a quark-anti-quark pair is produced during the final state radiation, we end up with more than one string. This process is however much less important than gluon radiation. In pp interactions most of the strings have still low masses, which is a direct consequence of parton distribution functions peaking at low $\mathrm{x}$. But the evolution of the tails is quite different. Whereas at $17.3 \mathrm{GeV}$ the distribution is steeply falling with almost no strings at all above 10 $\mathrm{GeV}$, the strings for pp at $200 \mathrm{GeV}$ reach much higher masses.

More conclusive is figure 6 where we see the corresponding cumulative distributions, i.e. the fraction

$$
F(m)=\frac{\int_{0}^{m} \frac{d n}{d m^{\prime}} d m^{\prime}}{\int_{0}^{\infty} \frac{d n}{d m^{\prime}} d m^{\prime}}
$$

of strings with masses below $m$. At $17.3 \mathrm{GeV}$ $50 \%$ of strings are lighter than $1 \mathrm{GeV}$, at 200 $\mathrm{GeV}$ the fraction is only $10 \%$. Strings below 1 $\mathrm{GeV}$ cannot produce any baryons. If we want to create a $\Omega$ given a $\mathrm{s}-\overline{\mathrm{d}}$ string, we will find in ad- 


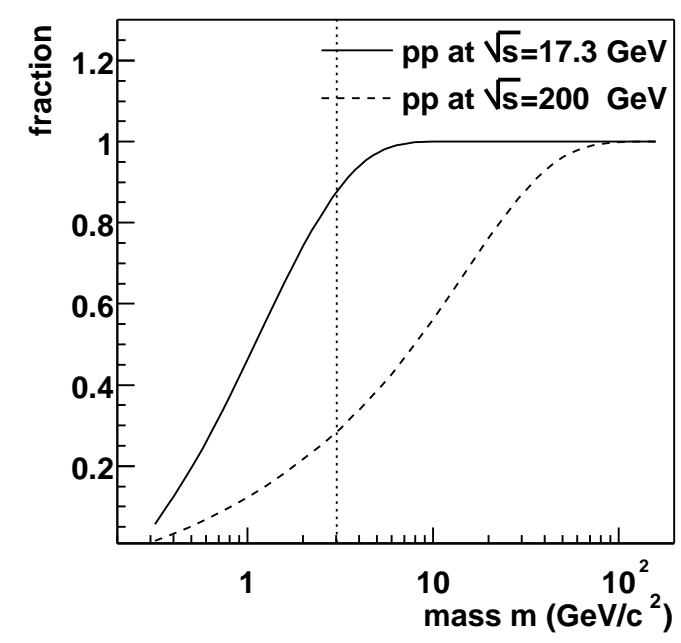

Figure 6: Integrated string masses: Shown is the relative fraction of strings below a certain mass $m$. The line at $3 \mathrm{GeV}$ shows the threshold for $\Omega$ production.

dition a $\bar{\Xi}$, since we have to break the string with the creation of a ss $-\overline{\mathrm{s}} \overline{\mathrm{s}}$ pair. Therefore the minimum mass is above $3 \mathrm{GeV}$ in the best case scenario, where one strange quark is already given by the initial string. Consequently, it is hard to create $\Omega$ 's at low beam energies since only $10 \%$ of the strings have the necessary mass, whereas at RHIC-energies $70 \%$ of the strings could kinematically produce $\Omega$ 's.

\section{Conclusions}

We can conclude that the different masses for the strings at the different beam energies are responsible for a possible suppression of heavy hadrons in pp collisions as compared to $\mathrm{e}^{+} \mathrm{e}^{-}$annihilation. If the hadron mass is small as compared to the typical string energy the hadron multiplicity ratios reach asymptotic values. A further increase of the string energy leads only to an overall increase of the produced hadron multiplicity leaving their relative ratio unchanged. If the string mass becomes comparable to the hadron masses, the production of these hadrons are suppressed due to the very limited phase space available.

This explains why in thermal fits [ [ mon constant temperature has been found for high energy $\mathrm{p} \overline{\mathrm{p}}$ collisions and $\mathrm{e}^{+} \mathrm{e}^{-}$annihilation. The physical origin of this phenomenon has in our model nothing to do with the formation of a thermal system. Therefore it is premature to identify this fit parameter with a true temperature. Employing a string fragmentation model which describes the kinematical variables as well the multiplicities of particle species in $\mathrm{e}^{+} \mathrm{e}^{-}$, pp

and $\mathrm{p} \overline{\mathrm{p}}$ collisions allows to interpret the results of [7] in physical terms without touching the claim that data can be well fitted using the functional forms of a grand canonical description.

\section{References}

[1] J. Rafelski, Phys.Rept.88:331,1982, Phys. Rev. Lett. 48:1066 (1982)

[2] F. Antinori et al., Nucl.Phys.A661:130 (1999)

[3] H.J. Drescher et al., hep-ph/0007198 accepted by Phys. Rep., Phys.Rev.Lett.86:3506 (2001)

[4] Opal collaboration: R. Ackers et al., Zeit.Phys. C63:181 (1994), G. Alexander et al., Zeit.Phys. C73:569 (1997), Zeit.Phys. C73:587 (1997)

[5] M. Gazdzicki, Nucl.Phys. A528:754 (1991)

[6] UA5 Collaboration, R.E. Ansorge et al., Nucl.Phys. B328:36 (1989)

[7] F. Beccattini, Z.Phys.C76:269 (1997), Z.Phys.C72:491 (1996) 